\title{
La conectividad en el sector español de carga aérea: 2004-2014
}

\section{Connectivity in the Spanish air freight transport sector: 2004-2014}

Núm. 9 (2017), pp. 113-138

Pais Montes, Carlos*

Freire Seoane, María Jesús**

González Laxe, Fernando***

Recibido: noviembre, 2016

Aceptado: diciembre, 2017

JEL Clasif: C45, D2, D85, L93

DOI: $10.5944 /$ reppp.9.2017.17590

\footnotetext{
*CARlos Pais Montes: Universidade da Coruña. E-mail:carlos.pais.montes@udc.es

**María Jesús Freire Seoane: Universidade da Coruña. E-mail: maje@udc.ess

***Fernando GonZÁlez Laxe: Universidade da Coruña. E-mail: laxe@udc.es
} 


\title{
Resumen
}

El segmento de las aerolíneas de carga, como demandante y usuario de servicios aeroportuarios, ha crecido en las dos últimas décadas hasta convertirse en parte fundamental de la red global de suministro en España. El objetivo de este artículo es analizar la evolución de la conectividad de este modo logístico, en el período 2004-2014, utilizando la base de datos del Observatorio para el Transporte y la Logística de España sobre posiciones aeroportuarias de carga de origen y destino. Los resultados indican que, en los mayores aeropuertos españoles (Madrid, Barcelona, Zaragoza y Vitoria), ha existido una recuperación del volumen de carga operado en los niveles pre-crisis, que no ha ido acompañada, no obstante, de una recuperación en términos de centralidad, con la excepción de Zaragoza, que es la infraestructura que muestra, actualmente, mayor resiliencia y mejores perspectivas en este aspecto..

Palabras clave: transporte aéreo; fletes aéreos; aeropuertos; logística; redes complejas; conectividad; teoría de grafos

\begin{abstract}
Air cargo transport segment in airlines, as a petitioner and user of airport services, has grown in the last two decades until being a key part of the global supply network in Spain. The goal of this article is to analyze the evolution of the connectivity of this logistic mode, along the 2004-2014 period, using the database of the Observatory for the Transport and the Logistics in Spain on airport cargo positions of arrival and departure.The results show that, in the biggest Spanish airports (Madrid, Barcelona, Zaragoza and Vitoria), it's been a recovering of the cargo throughput reaching pre-crisis levels, however this has not been accompanied by a recovering in terms of centrality, with the exception of Zaragoza, which stands as the only infrastructure currently showing more resilience and better perspectives in this aspect.
\end{abstract}

Key Words: air transport; air freights; airports; logistics; complex networks; connectivity; graph theory 


\section{Introducción}

La evolución de los diferentes modos de transporte, en términos de inversión y desempeño, constituye un elemento explicativo de los cambios operados en la economía global (Rietveld \& Bruinsma, 2012). En particular, el volumen total de carga aérea internacional representa todavía una proporción pequeña del volumen total mundial (1 \% en 2014), pero supone, en cambio, una parte importante en cuanto al valor de las mercancías transportadas (35\% del total) (IATA, 2016). Algunos autores han aproximado el impacto económico de la actividad logística de carga aerotransportada a nivel global, en un aumento del 2,4 \% del valor de la mercancía operada por cada punto de incremento en el PIB mundial, en las dos últimas décadas (Smyth \& Pearce, 2008), incidiendo, por tanto, en los ingresos tanto de las compañías aéreas como de los organismos de gestión aeroportuaria (Ducker, 2006; Escalona \& Ramos, 2010).

Todo ello pone de manifiesto la trascendencia de este modo de transporte, de importancia emergente en las economías desarrolladas (Coyle, 2002), y que registra perspectivas de crecimiento de un $4.7 \%$ interanual en la próxima década (Boeing, 2016).

El panorama logístico español también ha incorporado de modo muy significativo a la carga aérea como elemento central en lo que respecta a la capacidad exportadora/ importadora de las empresas nacionales (OTLE, 2016). La gran demanda por parte de empresas que tienen al comercio exterior como principal canal de operación, ha disparado la importancia jerárquica de algunos nodos logísticos, hasta el momento secundarios respecto a la cadena de suministro, como por ejemplo Zaragoza (Pastor, 2014; Escalona \& Ramos, 2010) o Vitoria (Ortega, 2015; Centro de Suministro, 2013); y ha complementado, además, el potencial de otros nodos multimodales como Las Palmas de Gran Canaria.

Pero, como ya ha quedado sólidamente establecido en lo relativo al transporte marítimo (Ducruet \& Notteboom, 2012; Pais-Montes et al., 2012), el análisis del desempeño de los nodos logísticos debe incluir, no solo el seguimiento estadístico del volumen total de mercancías movidas anualmente, sino también una cuantificación de la influencia que esas posiciones ostentan en la red global definida por la cadena de suministro.

El presente trabajo desarrolla, por primera vez, esta metodología, para la red de vínculos incidentes en los diferentes nodos de carga aérea españoles a lo largo del período 2004-2014, con el objetivo de determinar cuantitativamente la jerarquía aeroportuaria de carga, no solo en términos de throughput anual, sino también en lo que respecta a la influencia (centralidad) de esos mismos nodos.

Los resultados indican que, en los mayores aeropuertos españoles (Madrid, Barcelona, Zaragoza y Vitoria), ha existido una recuperación del volumen de carga operado en los niveles pre-crisis, que no ha ido acompañada, no obstante, de una recuperación en términos de centralidad, con la excepción de Zaragoza, que es la infraestructura que muestra, actualmente, mayor resiliencia y mejores perspectivas en este aspecto. 


\section{Antecedentes teóricos}

\subsection{La carga aerotransportada: aspectos operacionales}

Coyle (2002) afirma que el transporte aéreo se ha convertido en el sector de mayor crecimiento dentro del mercado de la carga, debido, fundamentalmente a la posibilidad de recorrer grandes distancias a velocidades considerables, a la gran escalabilidad en términos de capacidad de aumento de frecuencias y a las ratios de puntualidad. Estas tres características están directamente correlacionadas con los requisitos asociados a las pistas de aterrizaje, los equipamientos de las mismas y los requerimientos específicos de las operativas necesarias de trasvase intermodal. El control de estos elementos es clave a la hora de reducir los retrasos y los problemas de congestión (Wong y Wong, 2002). En este sentido, Pejovic et al. (2009) y Cook (2009) estiman que las demoras, sea cual sea la causa, implican muy elevados e imprevisibles costes asociados al consumo, a la tripulación, y a los costes marginales de mantenimiento. Las condiciones climatológicas adversas, según Abdelghany (2004), son el origen del $75 \%$ de los retrasos con respecto al schedule programado, y son causa de numerosas pérdidas en la capacidad máxima transportable, convirtiéndose, obviamente, en la mayor restricción de los aeropuertos (Schaefer y Millner, 2001).

En relación a la consecución de una estructura eficiente de costes, es preciso resaltar que los avances tecnológicos en aviónica, los nuevos procesos de fabricación y las economías de escala, han permitido una convergencia en términos de asequibilidad en precio y flete. $\mathrm{Y}$ aunque este aspecto, siga constituyendo una barrera para muchos cargadores, la reducción paulatina y continua de los costes, sumado al incremento de la calidad en el servicio, de la seguridad y de la frecuencia, permiten caracterizar a este modo de transporte como altamente competitivo con respecto al resto de opciones logísticas; de ahí la trascendencia de la elección de los centros de distribución de las aerolíneas (Martín y Román, 2004).

No son ajenos, tampoco, aquellos aspectos relacionados con las operaciones de control del tráfico aéreo, es decir, el número de aeronaves que se deben gestionar en un mismo espacio aéreo y los patrones de prioridad existentes en este campo (Dillinham, 2005). Dentro de la operativa técnica, Cao y Kanafani (2000) han estudiado la limitación de la capacidad de las pistas de aterrizaje y Takebayashi (2011) ha analizado la relación entre la dinámica de las aerolíneas logísticas en cuanto a las estrategias que siguen para maximizar capacidad de carga y pistas útiles.

La planificación de las terminales y su intersección con otros flujos de mercancías y con la tipología de los productos transportados, es decir, la especialización de las cargas, son también factores a tener en consideración; de la misma manera que lo constituyen las necesidades de almacenaje y consolidación de las cargas (Lee et al., 2006). En particular, la disponibilidad de contenedores aéreos es un elemento básico que contribuye a reducir los costes y a optimizar la capacidad de transporte de las aeronaves, contribuyendo, por tanto, a la consolidación de los centros de conexión ("hubs") (Wu, 2010; Lu \& Chen, 2010; Amaruchkul y Lorchirachoonkul, 2011).

Asimismo, es preciso tener en consideración la estrategia comercial de las aerolíneas. En este aspecto son muy clarificadores los trabajos de Mueller y Chatterji (2002) que 
analizan los errores de las compañías en sus tácticas operativas; o los de Wu (2005) que evalúa la desorganización estocástica de las compañías aéreas.

Rong y Grunow (2009) son los primeros investigadores que prestaron atención a las operaciones de carga aerotransportada desde un punto de vista de estrategia espacial. En su trabajo se postula que es necesario introducir cambios vinculados con la estructura de los operadores de tierra, en el sentido de deslindar de manera correcta tanto la organización del trabajo como la productividad de los operadores. En el mismo sentido, Schmitdberger et al. (2009) establecen que la competencia entre operadores de tierra, dentro de las terminales de carga, es necesaria y básica para lograr una mayor eficiencia y mejorar los niveles de competitividad. Chi-Lok y Zhang (2009) afirman que la ubicación de los aeropuertos posee un fuerte impacto en sus niveles de eficiencia, y que la existencia de uno o más competidores no debe ser considerada como problema, en la medida en que una mayor competencia contribuye a aumentar los flujos y a mejorar la eficiencia. En relación a la dimensión adecuada de los aeropuertos, Chow y Fung (2009) establecen que las economías de escala consiguen retroalimentar el transporte de pasajeros con el transporte de carga y viceversa, admitiendo que es posible generar flujos de carga asociados a los flujos de pasajeros; aunque es preciso determinar tanto los niveles de congestión de los propios aeropuertos y los niveles de rentabilidad de las inversiones efectuadas para ambos fines.

Por otro lado, la mejora de las informaciones entre agentes también fue objeto de tratamiento en relación a la productividad. Los estudios de Hsu et al. (2005) y de Chang y Wang (2010) buscan reivindicar la minimización del tiempo necesario en las operaciones de tierra y en implementar un sistema de monitorización de la carga, con objeto de dinamizar el flujo de información, mejorando la trazabilidad y el seguimiento de mercancías.

\subsection{La geografía internacional del transporte aéreo de carga}

El último informe sobre transporte aéreo del Banco Mundial muestra cómo la reactivación económica se traslada también a los niveles de tráficos aéreos, tanto en lo que respecta al número de pasajeros como al volumen de carga (World Bank Group, 2015), con una repercusión en el PIB mundial cercana al 3,4 \% y, además, con un gran impacto en los puestos de trabajo generados: 58,1 millones de empleos alrededor de 1.400 compañías aéreas y 4.000 aeropuertos.

A nivel microeconómico, se constata la creación de grandes alianzas entre las compañías aéreas de carga y la exigencia de aeropuertos con grandes zonas logísticas, con fuertes relaciones con las economías del entorno y capaces de integrarse en redes mundiales de servicios (Li et al., 2015; van Vliet, 2011; Gardiner et al., 2005).

Las principales rutas se estructuran, fundamentalmente, a lo largo de ejes horizontales situados en el hemisferio norte (figura 1), aunque, en el último decenio, emergen los flujos en el hemisferio sur y en el continente asiático (IATA, 2016). Teniendo en cuenta la carga total transportada en millones toneladas (MTm) y la distancia recorrida en Km entre el origen y el desino (Tm-Km), las mayores previsiones de crecimiento se corresponden con el comercio «Intra China» (10,9\%), «Medio Oriente-Europa» (9,5 \%) y «Europa-Nordeste Asia» (6,2 \%), mientras que los mayores retrocesos en la tasa de variación se esperan para Intra Norteamérica (del 9,1\% en 2001-2010 al -1,5 \% previsto para 2011-2020), EuropaNorteamérica (del 8,9 \% al 1,5 \%) y Asia-Norteamérica (del 20,3 \% al 4,3\%). 


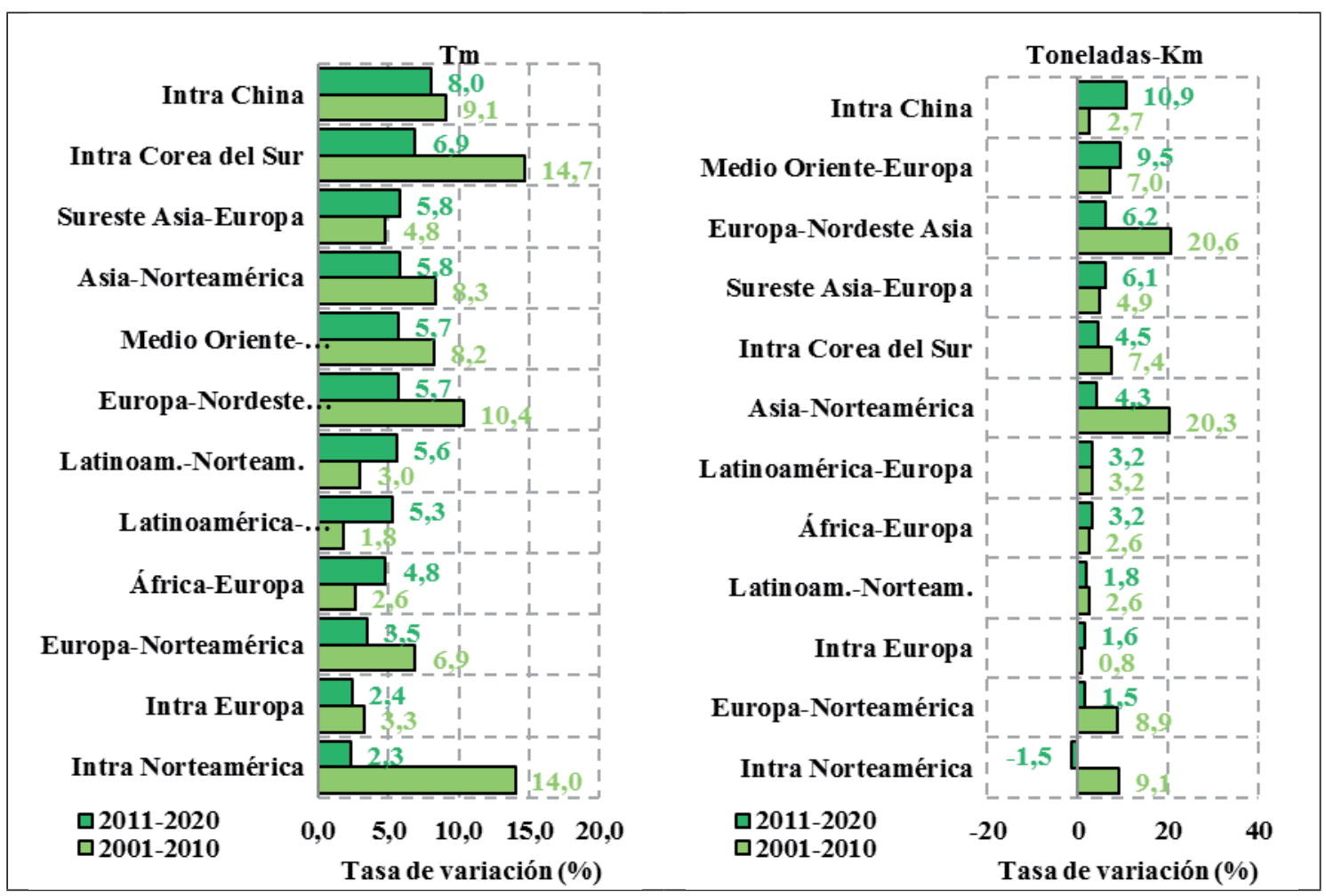

Figura I. Evolución del volumen de carga aerotransportada por zonas mundiales (200I-20l0) y previsión 20I l-2020.Tm y Tm-km. Ordenación según previsión 20 I I-2020.

Fuente: IATA (2016)

El transporte mundial de carga lo efectúan tanto compañías específicamente dedicadas a los servicios logísticos (FedEx, UPS, Korean Cargo, Cathay Pacific, Cargo Lux) como aerolíneas que, hasta el momento, han tenido al transporte de viajeros como línea principal de negocio (Lufthansa, KLM, Singapore Airlines, Emirates, China Airlines) (figura 2). Las compañías que más crecen entre 2005 y 2015, en términos de toneladas transportadas por Km recorridos (figura 2) son Qatar Airways (486 \%), Airbridge Cargo (418\%), China Southern (181\%), Polar Air Cargo (126 \%), Emirates (121\%), Etihad Airlines (121 \%) y All Nippon Cargo (104\%); mientras que en el primer cuartil de tasa de variación, es decir, en la zona de mayor decrecimiento de carga, se sitúan Air France (-44 \%), KLM (-25 \%), Singapore Airlines (-23\%), Eva Airlines (-21 \%), Korean Air (-19 \%), Lufthansa $(-17 \%)$ y China Airlines (-15\%). 


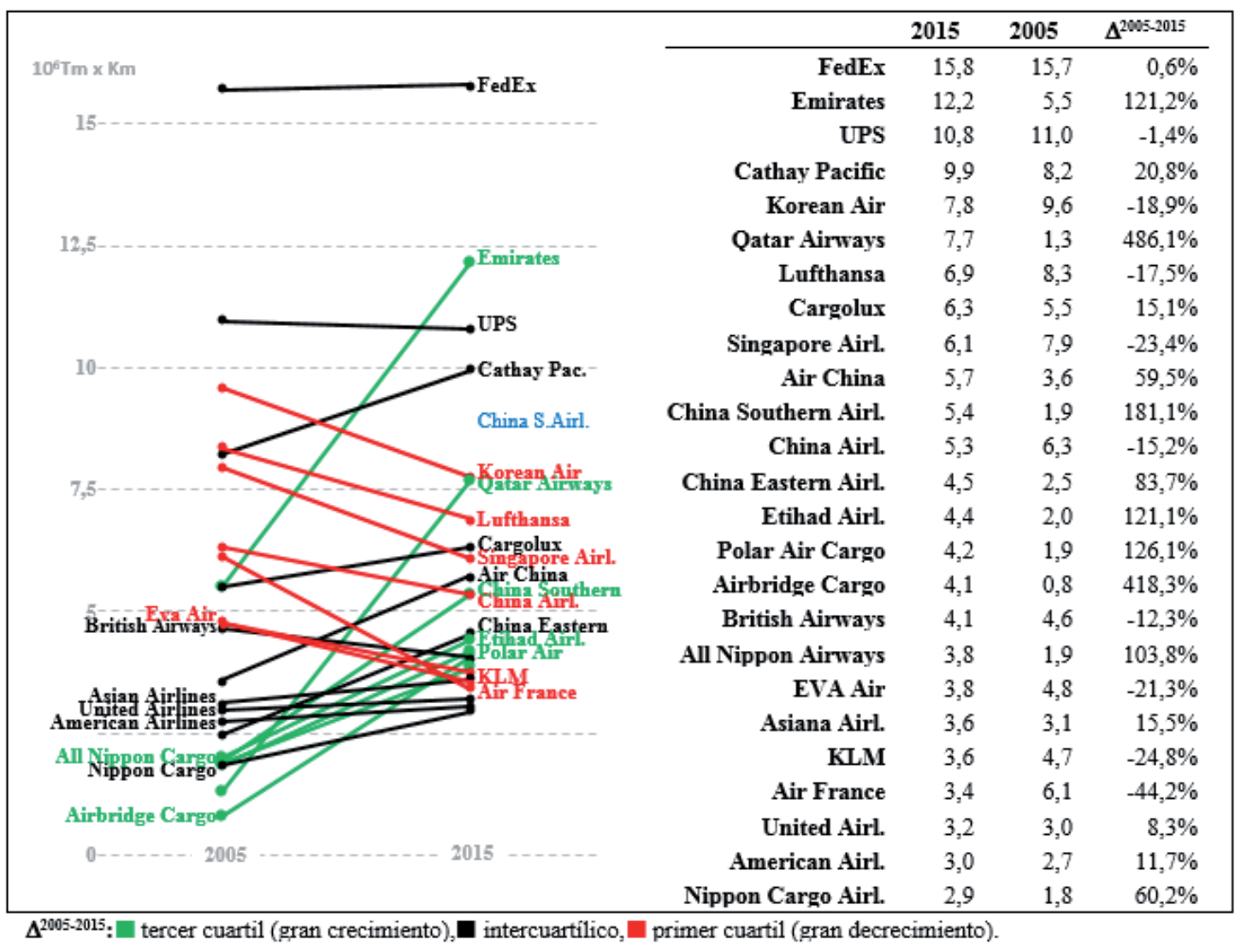

Figura 2. Ranking 2005-20I5 de las principales compañías aéreas de carga: Millones de Tm x Km. Fuente: IATA (2016).

En los aeropuertos asiáticos y europeos domina el tráfico de carga general, mientras que en los norteamericanos se ha incorporado el tráfico urgente y el courier internacional. La ubicación en Memphis (Tennesee, EEUU) de la compañía Federal Express -FedEx- y la localización en tres aeropuertos europeos (París, Frankfurt y Amsterdam) de la sede de los hubs de Air France, Lufthansa y KLM, respectivamente, son señales claras de la aparición de un fuerte proceso de concentración espacial de los flujos internacionales de carga aerotransportada.

Los niveles de especialización también son notables, como en los casos de la conurbación Colonia-Bonn (Alemania) y en East Midlands (Gran Bretaña) por sus importantes tráficos intra-europeos. Dicha especialización está bien motivada por los requisitos técnicos y tecnológicos a la hora de albergar y permitir la circulación de grandes aeronaves de carga, de realizar servicios con agilidad y puntualidad, y cumplir las normas de seguridad en las áreas de carga/descarga (Neibergeer, 2008).

Los cambios en las posiciones del ranking (figura 3) y su especialización vienen motivados por las organizaciones espaciales de los flujos de carga. Y estas se derivan de las dinámicas de inestabilidad de las propias redes productivas globales, y de las tensiones de competencia entre los diversos agentes que intervienen en la prestación de servicios de carga (Neibergeer, 2008). Los aeropuertos emergentes en términos de crecimiento del volumen de mercancías operado a lo largo del período 2005-2015 son Doha (que crece un $147 \%)$, Beijing (141\%), Guangzhou (104,933\%), Dubai (90 \%), Shanghai (76 \%), Shenzhen 
(58 \%), Abu Dhabi (53 \%), Tokyo-Haneda (46 \%) y Hong Kong (29 \%), es decir, exclusivamente, hubs chinos y de Oriente Medio. En cambio, los aeropuertos que se sitúan en el cuartil más bajo de tasa de crecimiento son New York-JFK (-22 \%), Newark (-21 \%), OsakaKansai (-12 \%), Indianápolis (-8 \%), Tokyo-Narita (-7 \%), Los Angeles (o \%), Anchorage (o \%), Atlanta (1\%), Singapore (1\%) y Chicago-O Hare (2 \%), es decir, fundamentalmente nodos estadounidenses.

Por último, las frecuentes reestructuraciones y los cambios en las compañías modifican el panorama. Las perspectivas siempre fueron optimistas (Clancy y Hoppin, 2006), salvo cuando se ven afectadas por otros factores extremos y complejos como los derivados del precio y disponibilidad del petróleo, fluctuaciones de las divisas, evolución de acuerdos bilaterales, políticas de cielo abierto, desequilibrios en los flujos del comercio internacional, inestabilidad política de ciertos países o regiones y aspectos vinculados al impacto medioambiental del transporte aéreo.

\begin{tabular}{|c|c|c|c|c|}
\hline \multirow{2}{*}{${ }^{10} \mathrm{Tm}$} & & 015 & 2005 & $\Delta^{1005-2015}$ \\
\hline & HongKong & 4,5 & 3,4 & $29,8 \%$ \\
\hline \multirow{10}{*}{ 4,5- - - - - - - - - - - - - $\overline{\text { pHoñg Kong }} \overline{-}$} & Memphis & 4,3 & 3,6 & $19,2 \%$ \\
\hline & Shanghai-Pudong & 3,3 & 1,9 & $76,3 \%$ \\
\hline & Anchorage & 2,6 & 2,6 & $0,8 \%$ \\
\hline & Seoul-Incheon & 2,6 & 2,1 & $20,8 \%$ \\
\hline & Dubai & 2,5 & 1,3 & $90,6 \%$ \\
\hline & Louisville & 2,4 & 1,8 & $29,5 \%$ \\
\hline & Tokyo-Narita & 2,1 & 2,3 & $-7,3 \%$ \\
\hline & Paris-DeGaulle & 2,1 & 1,8 & $18,1 \%$ \\
\hline & Frankfurt & 2,1 & 2,0 & $5,8 \%$ \\
\hline & Taipei & 2,0 & 1,7 & $18,5 \%$ \\
\hline anghai & Miami & 2,0 & 1,8 & $13,9 \%$ \\
\hline & Los Angeles & 1,9 & 1,9 & $0,5 \%$ \\
\hline & Beijing & 1,9 & 0,8 & $141,6 \%$ \\
\hline & Singapore & 1,9 & 1,9 & $1,8 \%$ \\
\hline & Amsterdam & 1,7 & 1,5 & $10,7 \%$ \\
\hline & Chicago 0 Hare & 1,6 & 1,5 & $2,9 \%$ \\
\hline & London Heathrow & 1,6 & 1,4 & $14,5 \%$ \\
\hline & Guangzhou & 1,5 & 0,8 & $104,9 \%$ \\
\hline & Doha & 1,5 & 0,6 & $147,1 \%$ \\
\hline & NewYork-JFK & 1,3 & 1,6 & $-22,2 \%$ \\
\hline & Bangkok & 1,2 & 1,1 & $7,9 \%$ \\
\hline nkft & Tokyo-Haneda & 1,2 & 0,8 & $46,8 \%$ \\
\hline & Shenzhen & 1,0 & 0,6 & $58,9 \%$ \\
\hline Paris-De C & Indianapolis & 1,0 & 1,1 & $-8,3 \%$ \\
\hline & AbuDhabi & 1,0 & 0,6 & $53,6 \%$ \\
\hline 0 & AlMaktoum-Dubai & 0,8 & 0,7 & $28,1 \%$ \\
\hline & Leipzig & 0,8 & 0,7 & $28,1 \%$ \\
\hline & Delhi & 0,8 & 0,7 & $16,4 \%$ \\
\hline & Istambul & 0,8 & 0,7 & $16,1 \%$ \\
\hline $\mathrm{ab}$ & Newark & 0,8 & 1,0 & $-21,6 \%$ \\
\hline & Osaka-Kansai & 0,8 & 0,9 & $-12,5 \%$ \\
\hline Stambul & Atlanta & 0,8 & 0,8 & $1,0 \%$ \\
\hline ballas- wroussels & Luxembourg & 0,8 & 0,7 & $4,3 \%$ \\
\hline Óaka-Kansai & Dallas-Ft.Worth & 0,8 & 0,7 & $7,9 \%$ \\
\hline $0,5----2005-$ & Brussels & 0,8 & 0,7 & $10,6 \%$ \\
\hline & Oakland & 0,8 & 0,7 & $15,8 \%$ \\
\hline
\end{tabular}

Figura 3. Ranking 2005-20I5 de los principales aeropuertos de carga del mundo: Millones de Tm. Fuente: IATA (20I6). 


\subsection{Características de la oferta y la demanda}

Las características de la oferta han contribuido a modificar la economía de las compañías aéreas históricas a favor de los operadores de bajo coste y de los transportistas del Golfo Pérsico. Estos nuevos actores han condicionado, en los últimos años, tanto a los aeropuertos regionales como a las plataformas $h u b$.

Por otro lado, la puesta en práctica de las denominadas «libertades aéreas», es decir, las dinámicas de liberalización de los servicios de transporte aéreo (Zhang \& Zhang, 2002), la emergencia de nuevos nichos de mercado, la nueva concepción logística de la cadena de suministro en el siglo XXI, y la puesta en marcha de la revolución low-cost, han contribuido no solo a la aparición de nuevos actores, sino también a democratizar el medio del transporte aéreo.

Entre las nuevas pautas de dicha reconversión, se encuentra la enorme demanda procedente de los nuevos países instalados en los Emiratos del Golfo, que han dirigido sus inversiones de cara a un posicionamiento en esta actividad con la idea de ubicarse en una situación geográfica ideal entre Europa y Asia. La creación de hubs, la prestación de servicios de calidad con tarifas competitivas, y la conversión en punto de escala cerca de los grandes centros comerciales, han acelerado su presencia en el top de la economía mundial.

\subsubsection{Funciones de oferta}

Los aspectos técnicos determinan las circunstancias y las posibilidades de transportar mercancías en las bodegas de los aviones o en los aviones cargueros específicos. Dependen, pues, de las características del aparato, de las distancias hasta el aeropuerto de destino, de las condiciones de la pista del aeropuerto de origen, y del peso máximo de despegue en función de las condiciones meteorológicas y la longitud de la pista.

Varias características de las funciones de oferta a corto plazo son: la «inamovibilidad» de las infraestructuras, que no permite aprovechar sus activos en ubicaciones distintas; las elevadas inversiones de capital inicial para la puesta en marcha; y la indivisibilidad de sus activos, que revela la presencia de costes hundidos.

La presencia de barreras de entrada, tanto de carácter estructural (orografía, climatología) como legales (limitaciones urbanísticas) o medioambientales (presencia próxima de hábitats naturales o espacios urbanos) limitan su implantación.

La proporción de costes fijos es elevada, próxima al 90 \% (Thelle, 2012), lo que condiciona la operatividad económica de los mismos. Se necesitan economías de escala y existe un amplio debate en torno a los umbrales mínimos y la existencia de las des-economías externas (Müller et al., 2005; Forsyth, 2010; Lechmann, 2011).

Desde la perspectiva del transporte aéreo las compañías operan en forma de redes complejas, basadas en el concepto hub\&spoke (Bowen, 2012). Es decir, existe un reducido número de nodos con muchas conexiones directas, y una amplia población de nodos secundarios con un número bajo de conexiones. Las rutas de alimentación son cortas y se construyen en torno a los hubs más conectados.

Las compañías operan con sus propias flotas de aviones, aunque también se dan casos de chartering y la coexistencia de aviones cargueros puros con aviones donde las mercancías se combinan con las operaciones mixtas pasajero-carga, en el que la carga es un subproducto del transporte en la ruta predeterminada. 


\subsubsection{Funciones de demanda}

Las funciones de demanda están sujetas a circunstancias específicas y a características propias de esta modalidad logística: a) la carga aérea no regresa, por lo que existen unos desequilibrios direccionales en las rutas, que pueden llegar a ser significativos; b) las cargas pueden ser muy heterogéneas, de distinta naturaleza, volumetría, densidad y seguridad; c) la existencia de servicios competitivos por parte de otros transportes alternativos (tráfico rodado y transporte marítimo).

Ya se ha visto, además, cómo la demanda de transporte aéreo de carga está relacionada con el crecimiento económico internacional, con los precios del combustible, y con los propios patrones de la cadena de suministro. Del mismo modo, existen unos costes de oportunidad logística, que se traducen en la cuantificación de las ventajas competitivas con respecto a otros modos de transporte.

Tradicionalmente, los aeropuertos han sido considerados como infraestructuras fijas en donde, aerolíneas por un lado, y empresas de servicios, por otro, desarrollan modelos de negocio distintos y a la vez complementarios. Los aeropuertos tratan de satisfacer a los clientes, que están integrados en cadenas de suministro globales, con lo que se obtiene, pues, una verticalidad procedimental en la estos tratan de prestar su servicio de manera distinta a las aerolíneas, y estas a los consumidores. Este fenómeno se denomina two-sided markets, o mercados multiplataforma (CNMC, 2014). En ellos, el volumen de transacciones depende de ciertos factores relacionados con la interdependencia de ambos lados (vínculo reticular bidireccional entre los nodos). Los aeropuertos se sitúan en una posición intermedia entre los grupos de clientes y los prestatarios de servicios, y sus perspectivas están delimitadas por: las posibilidades de negocio vinculadas al logro de rentabilidades; el aumento de la cuota de mercado y la eficiencia; y, por último, por la utilización positiva de las infraestructuras y equipamientos. En suma, el sector aeroportuario se enfrenta a una demanda dual e interdependiente: la compuesta por las aerolíneas y la formada por los pasajeros.

\section{Metodología}

Las perspectivas contemporáneas sobre el estudio de la evolución de la carga transportada por cualquier medio logístico, no solo tienen en cuenta la variación en el volumen operado por un nodo a lo largo de un determinado período temporal, sino que añaden una perspectiva bidimensional que incluye las relaciones que establecen entre sí los nodos, es decir un análisis de redes complejas (Hesse y Rodrigue, 2004). Esto posibilita la delimitación de jerarquías nodales no solo en términos de volumen anual, sino también en lo que respecta a la influencia local/regional/global del nodo logístico, que podría ser muy distinta a lo que su rango en términos de total throughput pudiera estar sugiriendo (Pais-Montes et al., 2012).

En el caso concreto de las redes comerciales de transporte aéreo de mercancías, se dispone a través de OTLE (2016b) del total cargado y descargado en cada nodo $\mathrm{x}_{\mathrm{i}}$, así como de los nodos de procedencia y de destino, para el período 2004-2014. Esto posibilita, por tanto, la construcción de grafos dirigidos $\mathrm{G}^{\mathrm{t}}$ en base a los datos de oferta y demanda proporcionados por OTLE (2016b) para cada año, que incluyen vínculos dirigidos $\overrightarrow{\left(\mathrm{x}_{\mathrm{i}}, \mathrm{x}_{\mathrm{j}}\right)}$ de dos naturalezas distintas [1]: aeropuertos españoles (46 nodos $\mathrm{x}_{\mathrm{i}}^{\text {esp }}$ ) 
y agregados geográficos internacionales (9 nodos $x_{i}^{\text {agr: }}$ Unión Europea Schengen -UE1-, Unión Europea no Schengen -UE2-, Europa no Unión Europea Schengen -EU1-, Europa no Unión Europea no Schengen -UE1-, Africa -AF-, América del Norte -AN-, América Latina y Caribe -AL-, Asia y Pacífico -AS-, y Oriente Medio -OR-) [2]. El peso de cada nodo representa las Tm cargadas y descargadas anualmente, mientras que el peso del vínculo dirigido representa las Tm que se transportan de un nodo a otro.

Con esta configuración inicial, queda posibilitado un análisis de la conectividad para cada nodo basado en los siguientes parámetros:

a) Grado ("Gra", "Degree Centrality"): número de vínculos incidentes (dirección de llegada o salida) en un nodo, i.e., dada la matriz de adyacencia $A\left(G^{t}\right)_{i j}$, $\operatorname{grado}\left(\mathrm{x}_{\mathrm{i}}\right)=\sum_{\mathrm{i} .} \mathrm{A}\left(\mathrm{G}^{\mathrm{t}}\right)_{\mathrm{i} .}+\sum_{\mathrm{i}} \mathrm{A}\left(\mathrm{G}^{\mathrm{t}}\right)_{\mathrm{i}}$

b) Cercanía ("Cer", "Closeness Centrality"): inversa del promedio de todas las distancias geodésicas -distancias más cortas entre ese y otro nodo arbitrario de la red(Newman, 2008). Cuanto más grande es este parámetro, más corta es la distancia entre ese nodo y cualquier otro nodo arbitrario y, por tanto Cer puede ser interpretado como un indicador de lo eficiente que es un nodo a la hora de distribuir mercancía hacia otros nodos (Okamoto et al., 2008).

c) Intermediación ("Inf”, "Betweenness Centrality”): suma de las proporciones con las que un nodo intermedia a rutas arbitrarias pasando por ese nodo (Brandes, 2008), i.e. $\operatorname{Int}\left(\mathrm{x}_{\mathrm{i}}\right)=\sum_{\mathrm{x}_{\mathrm{j}}, \mathrm{x}_{\mathrm{k}}} \#\left\{\overrightarrow{\text { geodésica }\left(\mathrm{x}_{\mathrm{j}}, \mathrm{x}_{\left.\mathrm{i}, \mathrm{x}_{\mathrm{k}}\right)}\right)} / \# \overrightarrow{\text { geodésica }\left(\mathrm{x}_{\mathrm{j},}, \mathrm{x}_{\mathrm{k}}\right)}\right.$.

El parámetro es, por tanto, una medida de la influencia que el nodo $x_{i}$ ejerce sobre el flujo de información, en el sentido de que una petición arbitraria de origen-destino tiene más probabilidades de pasar por $\mathrm{x}_{\mathrm{i}}$ cuanta más Inf tenga (Newman, 2008).

d) Centralidad de autovalor (“Autv", "Eigenvector Centrality"): el mayor autovalor del espacio de autovectores de la matriz de adyacencia (Bonacich, 2007), i.e. $\operatorname{Autv}\left(\mathrm{x}_{\mathrm{i}}\right)=\max _{\lambda}\left\{\lambda \in R\right.$ t.q. $\left.\lambda \mathrm{x}_{\mathrm{i}}=\sum_{\mathrm{j}} \mathrm{A}\left(\mathrm{G}^{\mathrm{t}}\right)_{\mathrm{ij}} \mathrm{x}_{\mathrm{j}}\right\}$.

Esta definición puede ser interpretada como una suma ponderada de las posiciones que se conectan con a nodo, donde las rutas de mayor longitud son penalizadas con pesos menores. Por lo tanto, esta medida permite complementar la información obtenida a través de la centralidad de grado, cercanía e intermediación, con la caracterización de una nueva jerarquía, útil para discriminar si esa centralidad se produce con respecto a nodos influyentes o de menor importancia (Bonacich, 2007; Ruhnau, 2000; Franceschet, 2014).

La estructura de conectividad de cada red $\mathrm{G}^{\mathrm{t}}$ puede ser caracterizada a nivel global, además de por los estimadores puntuales usuales de la media y el Índice de Gini, por los siguientes indicadores adicionales: densidad del grafo, o razón entre el número real y el máximo de aristas posibles (Johns, 2012); modularidad, o fracción de aristas que pueden ser agrupadas en un número determinado de sub-grafos (Newman, 2006), i.e., número de comunidades de sub-grafos con alta conexión entre sus nodos y baja conectividad intercomunitaria; diámetro, o mayor distancia entre dos nodos arbitrarios de la red (Biskup, 2011); coeficiente promedio de clusterización (Average Clustering Coefficient), o promedio de la fracción de vecinos conectados para cada nodo, lo que indica, por tanto, la cohesión local de cada nodo (Barrat et al., 2004); y Longitud Media de Camino (Average Path Length), o distancia promedio entre todos los puntos del nodo (Chen et al., 2008). 
La posterior transformación de los grafos $\mathrm{G}^{\mathrm{t}}$ calculados para cada año en árboles mínimos de expansión $\hat{G}^{t}$ (Yaman et al., 2001) posibilita el cálculo de rutas de coste mínimo. Éstas simulan la perspectiva de un ficticio operador logístico global, que actúa exclusivamente sobre el sistema de carga aeroportuaria español, y que necesitaría planificar una estructura inicial óptima, en cuanto a los costes del transporte, para el año en que la operación comienza. Para la estimación de los costes logísticos entre dos nodos, se puede considerar a la inversa del volumen total de carga transportada entre esas dos posiciones como una aproximación funcional válida del coste del transporte, siguiendo las indicaciones de Limão y Venables (2001), Zohil y Prijon (1999) y Bougheas et al. (1999).

Por otro lado, a falta de una concreción computacional fiable para el algoritmo ChuLiu-Edmonds (McDonald et al., 2005; Clerot, 2015), que calcula el árbol mínimo de expansión en grafos dirigidos, y dado que se verifica el test $\mathrm{Chi}^{2}$ de cuasi-simetría para la matriz de adyacencia (Sharp y Markham, 2000), es decir, que el volumen de mercancía cargada es de magnitud similar al de mercancía descargada; se puede optar por el algoritmo clásico de Kruskal para grafos no dirigidos (Kruskal, 1956) utilizando la herramienta Tulip (Bourqui et al., 2008).

El árbol mínimo de expansión para cada año representa, en consecuencia, la estructura reticular óptima para la red de transporte aéreo de mercancías en España y posibilita la interpretación global del sistema logístico en términos de nodos influyentes y nodos periféricos, no solo desde el punto de vista de la conectividad, sino también en lo que respecta a la optimalidad de la estructura de costes sugerida por los vínculos privilegiados por el algoritmo de Kruskal.

\section{Conectividad y estructura mínima de costes}

La tabla 1 presenta los resultados obtenidos al calcular las tres redes de transporte aéreo de mercancías, para los 46 nodos españoles y los 9 nodos agregados que contienen la información de conectividad de los aeropuertos españoles con otros países, de acuerdo con la información disponible en OTLE (2016) (el significado de las abreviaturas figura en el Anexo 1).

Es importante destacar que los nodos con un volumen de mercancías menor a 0,1MTm en 2014 han sido excluidos del cuadro pero no del análisis de red. Los aeropuertos se pueden clasificar por orden decreciente de magnitud de carga: GRO (o,o9MTm); LCG (0,08); OVD y VDE (0,07); VLL (0,02); PNA, LEI, ABC y XRY (0,01); y, por último, con menos de o,o1MTm GRX, RGS, SDR, EAS, GMZ, REU, MJV, BJZ, RJL, LEN, SLM, JCU, TOJ, MCV, QSA Y AEI. También es importante señalar que en el cuadro tampoco aparecen los agregados internacionales: han sido tenidos en cuenta para la construcción de la red, como elementos para los que OTLE (2016b) define conexiones válidas de origen/destino con los nodos españoles, pero sus parámetros individuales de conectividad y de volumen de carga operada (.UE1 con 156MTm, .AL con 112, .OR con 94, .AN con 87, .AS con 41, .EU2 con 35, .UE2 con 23, .AF con 11 y .EU1 con 5) no pueden ser tenidos en cuenta en el análisis debido a la imposibilidad formal de determinar la interrelación entre ellos.

El análisis de los parámetros globales de red en los tres años analizados (2004, 2009 y 2014) arroja una visión dinámica de la evolución general del sistema aeroportuario de 
carga español. Atendiendo, en primera instancia, al volumen promedio anual de carga, se observa cómo la Gran Recesión tuvo un impacto importante, pasando de 18,8MTm en 2004 a 17,7 en 2009, recuperando posteriormente niveles superiores a los 20MTm en 2014. El estancamiento del indicador de Gini en el período 2004-2014 sugiere que ese aumento en el volumen promedio de mercancías no se ha llevado a cabo en terminales aeroportuarias emergentes, sino en otras con actividad ya consolidada.

Los nodos más importantes (MAD, BCN, ZAZ) experimentan (al contrario que el resto de aeropuertos con más de $0,1 \mathrm{MTm}$ operadas) una mejoría en el volumen de carga operada, pero esto no se traduce en una mejora de la conectividad en las terminales aeroportuarias españolas, lo que puede estar indicando problemas de ineficiencia en las infraestructuras existentes. De este modo, el grado promedio (conectividad directa) se mantiene en 20 para el 2004 y el 2009, pero cae drásticamente hasta 16 unidades en 2014, lo que quiere decir que los nodos aéreos de mercancías españoles han perdido vínculos en una media de 4 conexiones comerciales (-2,1 \% de variación interanual). Además, en el resto de estimadores de centralidad se producen caídas en el indicador: $-0,9 \%$ en la cercanía, $-1,5 \%$ en la influencia y $-1,5 \%$ en la centralidad de autovalor. Esta pérdida de conectividad generalizada en el sistema aeroportuario español se traduce en el descenso de la clusterización promedio (que pasa de 0,655 en 2009 a 0,504 en 2014), lo que indica una red menos cohesionada; y en la proliferación de sub-comunidades (4 en 2004, 7 en 2009 y 10 en 2014) lo que refuerza la hipótesis de la pérdida generalizada de relevancia de los principales hubs de carga aérea españoles.

En cuanto al comportamiento concreto de los principales nodos en lo que respecta a la evolución de su centralidad: MAD (365MTm en 2014) reduce su conectividad directa en un -0,6 \% (bajando desde 82 unidades en 2004 a 77 en 2014 -i.e. pérdida de conexiones directas con 5 nodos-), aunque consigue mejorar su capacidad de intermediación (Inf pasa de 532 unidades en 2004 a 581 en 2014), lo que indica que si bien la capacidad de conexión con los nodos más cercanos se ha visto mermada, desde el punto de vista comercial, la influencia con respecto a posiciones más lejanas ha aumentado ligeramente; BCN (102MTm en 2014) muestra, en cambio, una pérdida de centralidad desde todos los puntos de vista, i.e., pierde conexiones directas, pierde cercanía y capacidad de intermediar rutas regulares; el nodo de ZAZ (86MTm en 2014), principal hub de carga emergente a lo largo del período 2004-2014, es el único que muestra una evolución positiva en los principales indicadores de centralidad excepto en el autovalor $(-3,1 \%$, el valor más bajo de la serie para 2014), lo que no es necesariamente negativo, pudiendo ser interpretado como una evolución muy positiva en términos de expansión de su área comercial de influencia, con una preferencia para dirigir el negocio hacia nodos todavía no demasiado saturados y alejarse de los aeropuertos más transitados (lo que constituye una ventaja en términos de la falta de operadores competidores en esos hubs); por último, el nodo VIT, cuarta posición aeroportuaria en volumen de mercancías movidas, muestra una disminución en todos los parámetros de conectividad, especialmente acusada en el parámetro de autovalor, lo que ha de ser interpretado inequívocamente como una señal de que se ha perdido, de modo brusco, una línea de negocio que, hasta el momento, era vital para los resultados de este aeropuerto.

Es posible mencionar, no obstante, algún caso de éxito para el sistema analizado, en puertos con menor volumen de operaciones: IBZ (2,6MTm en 2014) que muestra, a pesar de la caída en el throughput del -3,6 \%, una gran agilidad a la hora de captar/distribuir 
mercancía de/hacia nuevos clientes, con el mayor aumento interanual del grado $(1,1 \%)$ y el segundo mayor aumento de la centralidad de autovalor (o,8 \%), lo que implica que esa mejora en la conectividad se dirige, además, hacia aeropuertos influyentes; FUE (o,9MTm en 2014) que consigue evoluciones muy favorables en la centralidad, sobre todo en la influencia (su capacidad de intermediar entre otras rutas de nodos importantes $-1,5 \%$ de crecimiento en la centralidad de autovalor- se ha potenciado desde las 0,3 unidades en 2004 hasta 2,2 en 2014). Es preciso, no obstante, introducir en esta evaluación positiva, la condición de posiciones insulares y, por tanto, la elevada dependencia de este tipo de transporte para estas demarcaciones geográficas [8].

*Ver Tabla I en la siguiente página 
Tabla I. Parámetros de conectividad para la red española de transporte aéreo de mercancías ordenados por MTm en 20I4, nodos con más de 0,IMTm operadas en 20I4). Fuente: OTLE (2014) y elaboración propia.

\begin{tabular}{|c|c|c|c|c|c|c|c|c|c|c|c|c|c|c|c|c|c|c|c|c|}
\hline & \multicolumn{5}{|c|}{2004} & \multicolumn{5}{|c|}{2009} & \multicolumn{5}{|c|}{2014} & \multicolumn{5}{|c|}{$\operatorname{VAR}(2004,20 \mid 4)$} \\
\hline & MTm & Gra & Cer & Inf & Autv & MTm & Gra & Cer & Inf & Autv & MTm & Gra & Cer & Inf & Autv & MTm & Gra & Cer & Inf & Autv \\
\hline$A D$ & 340,0 & 82 & 0,84 & 532,6 & 1,00 & 302,6 & 83 & 0,88 & 489,0 & 1,00 & 365,3 & 77 & 0,88 & 581,1 & 1,00 & 0,7 & $-0,6$ & 0,5 & 0,9 & 0,0 \\
\hline CN & 86,7 & 75 & 0,80 & 427,0 & 0,94 & 91,5 & 78 & 0,89 & 361,3 & 0,94 & 102,7 & 64 & 0,78 & 255,7 & 0,93 & 1,9 & $-1,5$ & $-0,3$ & $-4,0$ & 0,0 \\
\hline ZAZ & 9,3 & 22 & 0,50 & 5,2 & 0,54 & 36,9 & 30 & 0,56 & 16,8 & 0,62 & 86,6 & 23 & 0,56 & 18,6 & 0,37 & 83,5 & 0,5 & $\mathrm{I}, \mathrm{I}$ & 25,7 & $-3,1$ \\
\hline VIT & 44,0 & 32 & 0,57 & 18,6 & 0,58 & 27,4 & 34 & 0,59 & 29,1 & 0,54 & 41,9 & 25 & 0,57 & 17,6 & 0,45 & $-0,5$ & $-2,2$ & $-0,1$ & $-0,5$ & $-2,3$ \\
\hline LPA & 40,8 & 44 & 0,63 & $|3|, 8$ & 0,71 & 28,5 & 47 & 0,65 & 111,0 & 0,76 & 24,6 & 37 & 0,62 & 68,1 & 0,66 & $-4,0$ & $-1,6$ & $-0,1$ & $-4,8$ & $-0,7$ \\
\hline TFN & 24,6 & 32 & 0,58 & 83,1 & 0,52 & 15,4 & 42 & 0,67 & 88,4 & 0,52 & 13,7 & 29 & 0,58 & 123,3 & 0,53 & $-4,4$ & $-0,9$ & $-0,2$ & 4,8 & 0,2 \\
\hline VLC & 11,0 & 45 & 0,65 & 67,2 & 0,69 & 10,9 & 52 & 0,71 & 87,5 & 0,77 & 12,8 & 40 & 0,64 & 44,3 & 0,70 & 1,6 & $-I, I$ & $-0,2$ & $-3,4$ & 0,2 \\
\hline PMI & 21,4 & 56 & 0,72 & 135,9 & 0,75 & 17 & 51 & 0,67 & 85,6 & 0,80 & 11,3 & 40 & 0,66 & 50,6 & 0,66 & $-4,7$ & 9 & $-0,9$ & $-6,3$ & $-1,3$ \\
\hline sve & 4 , & 37 & 0,61 & 33,6 & 0,63 & -5 & 37 & 0,62 & 23,6 & 0,57 & 5,6 & 28 & 0,57 & 15,5 & 0,58 & I,8 & $-2,4$ & $-0,7$ & $-5,4$ & $-0,7$ \\
\hline TFS & 9,5 & 4 & 0,60 & 31,1 & 0,71 & 5,4 & 37 & 0,62 & 13,7 & 0,65 & 3,4 & 30 & 0,58 & 32,8 & 0,60 & $-6,4$ & $-2,5$ & $-0,5$ & 0,5 & $-1,4$ \\
\hline BIO & 4 , & 3 & 0,62 & 23,2 & 0,61 & & 33 & 0,60 & 9,6 & 0,60 & 2,9 & 29 & 0,59 & 15,2 & 0,58 & $-3,3$ & 9 & $-0,5$ & 4 & $-0,4$ \\
\hline ALC & 5 , & 3 & 0,58 & 10,0 & 0,57 & & 30 & 0,58 & 10,6 & 0,53 & 2,7 & 26 & 0,59 & 8,4 & 0,50 & $-5,5$ & 6 & , & $-1,6$ & $-1,3$ \\
\hline IBZ & $4, C$ & 18 & 0,52 & 2,0 & 0,40 & & 20 & 0,55 & 2,0 & 0,37 & 2 & 20 & 0,55 & 3,0 & 0,43 & $-3,6$ & I,I & 0,5 & 4, & 0,8 \\
\hline AGP & 6,7 & 44 & 0,66 & 195,2 & 0,64 & 3,5 & 42 & 0,64 & 137,2 & 0,66 & 2,5 & 32 & 0,61 & 82,8 & 0,54 & $-6,3$ & $-2,7$ & $-0,7$ & $-5,8$ & $-1,6$ \\
\hline AH & 3,4 & 18 & 0,53 & 46,4 & 0,39 & 2,6 & 17 & 0,53 & 0,5 & 0,37 & 2,3 & 15 & 0,53 & 0,4 & 0,34 & $-3,2$ & $-1,7$ & 0,2 & $-9,9$ & $-1,2$ \\
\hline scQ & 4,8 & 26 & 0,55 & 3,2 & 0,54 & 2,0 & 28 & 0,57 & 6,8 & 0,52 & 2,1 & 25 & 0,55 & 6,0 & 0,53 & $-5,6$ & $-0,4$ & 0,0 & 8,6 & $-0,2$ \\
\hline ACE & 7,6 & 26 & 0,55 & 7,3 & 0,53 & 4, & 34 & 0,61 & 13,5 & 0,53 & 2,0 & 26 & 0,58 & 10,3 & 0,53 & -7 & 0,0 & 0,6 & 4,1 & 0,1 \\
\hline FUE & 3,6 & 15 & 0,51 & ,3 & 0,35 & 1,9 & 24 & 0,56 & 3,6 & 0,47 & 0,9 & 16 & 0,54 & 2,2 & 0,40 & -7 & 0,7 & 0,6 & 76,9 & I,5 \\
\hline $\mathbf{s}$ & 1,6 & 15 & 0,52 & 13,4 & 0,27 & I,I & 17 & 0,53 & 1,2 & 0,33 & 0,6 & 10 & 0,53 & I,I & 0,19 & $-6,6$ & $-3,3$ & 0,3 & $-9,2$ & $-2,8$ \\
\hline VGO & 1,0 & 22 & 0,53 & 5,1 & 0,43 & 0,8 & 24 & 0,54 & 10,3 & 0,52 & 0,5 & 15 & 0,53 & 8,1 & 0,32 & $-5,7$ & $-3,2$ & 0,0 & 5,8 & $-2,6$ \\
\hline MLN & 0,4 & 8 & 0,48 & 2,3 & 0,12 & 0, & 6 & 0,49 & 0,5 & 0,14 & 0,1 & 4 & 0,48 & 0,0 & 0,10 & $-6,6$ & $-5,0$ & 0,0 & $-10,0$ & $-1,9$ \\
\hline
\end{tabular}

\section{Parámetros globales de red}

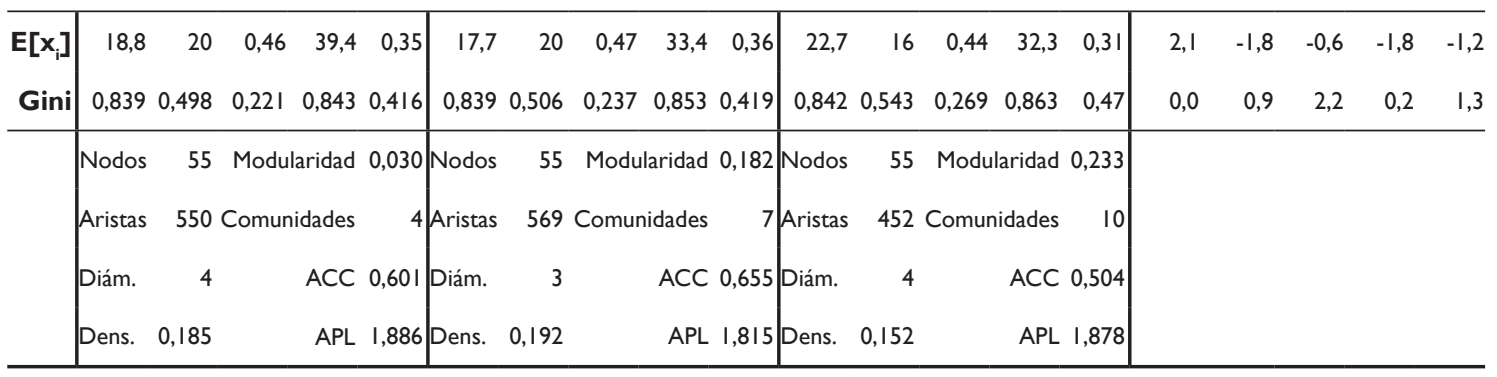

$>$ Percentil ${ }^{95}, \quad>$ Percentil ${ }^{85} \quad<$ Percentil ${ }^{15}$, Dens. $=$ «Densidad», Diám. $=«$ Diámetro», $A C C=«$ Coeficiente medio de clusterización (Average Clustering Coefficient)», APL=«Longitud media de camino (Average Path Length)». 

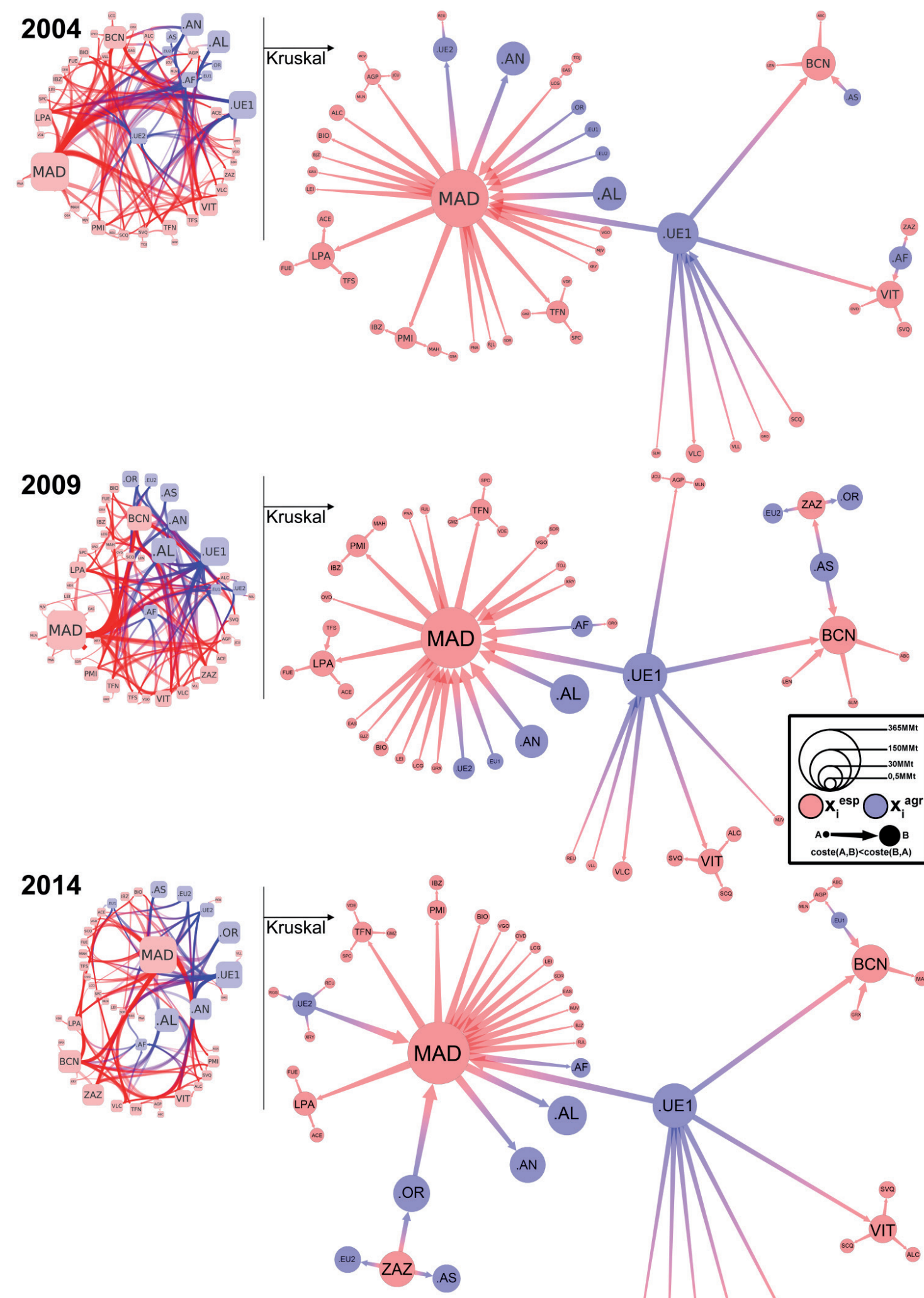
La figura 4 muestra la visualización de las tres redes construidas para el análisis. Se parte, como se ha indicado en la metodología, de la red general de 55 nodos y 452-550 aristas construida con los datos de OTLE (2014) para cada año, utilizando una metodología force-directed o de separación (Holten y Van Wijk, 2009) de tipo Balloon-OGDF (Lin y Yen, 2007; Carriere y Kazman, 1995). Para cada estructura global se calcula el sub-grafo resultante de la aplicación del algoritmo de Kruskal utilizando Tulip (Lambert y Aubert, 2012), obteniendo un recubrimiento para todos los nodos que minimiza los costes del transporte entre dos nodos. El algoritmo permite, además, discriminar la dirección de la arista cuyo coste es menor.

Con la información disponible se pueden realizar dos tipos de consideraciones: una con respecto a la estructura global de red y otra con respecto a la evolución de los subgrafos obtenidos a través de la aplicación del algoritmo de Kruskal.

Atendiendo a las estructuras globales de red, se pueden observar los siguientes comportamientos:

- MAD se constituye como hub de reparto prioritario únicamente en 2014, atrayendo gran parte de la actividad internacional, en detrimento de $\mathrm{BCN}$, aeropuerto sometido a una severa caída en la conectividad que pierde el sólido clúster consolidado a lo largo de 2009.

- .AF, a pesar de su todavía discreto indicador de throughput, consigue situarse en posiciones centrales en la red, tanto en 2009 como en 2014, atrayendo tráfico de multitud de nodos situados en diferentes posiciones.

- AGP ha pasado de ocupar una posición central con respecto a un amplio clúster en 2004, a situarse en posiciones periféricas de baja conectividad en 2009 y 2014.

Los sub-grafos obtenidos a través de la aplicación del algoritmo de Kruskal a las estructuras de separación permiten afirmar que:

- La arista MAD-.UE1 sigue siendo el eje sobre el que se sostiene y articula la actividad completa de la red logística en su conjunto. La estructura de costes es más favorable para las importaciones desde .UE1 que para las exportaciones hacia ese agregado.

- BCN pierde el vínculo con .AS que mantenía en 2004 (de modo exclusivo) y en 2009 (donde ya empieza a compartirlo con ZAZ). En 2014, el aeropuerto de $\mathrm{BCN}$ ya solo es relevante a nivel .EU1 e Islas Baleares.

- ZAZ adquiere un estatus de clúster independiente del resto de nodos de la red centralizando el abastecimiento procedente de/dirigido desde .OR, .EU2 y .AS. La dirección de los vínculos del clúster zaragozano sugiere una muy favorable configuración de costes para la actividad de exportación con origen en ZAZ.

- A pesar de haber perdido su conexión con .AF (crisis de las importaciones pesqueras), el aeropuerto de VIT mantiene su papel de hub distribuidor entre .UE1 y SVQ-SCQ-ALC.

- AN y .AL siguen privilegiando las conexiones con MAD, pero en 2004 la estructura de costes era favorable a las exportaciones a .AN e importaciones desde .AL; en 2009 los costes son más favorables para las operaciones de importación; y en 2014, en cambio, los vínculos más rentables son los de exportación desde MAD. 


\section{Conclusiones}

El segmento de las aerolíneas de carga, como demandante y usuario de servicios aeroportuarios, ha crecido en las dos últimas décadas hasta convertirse en parte fundamental de la red global de suministro.

Análogamente a otros modos de transporte, la actividad de carga aérea tiene un impacto positivo sobre las variables macroeconómicas de su zona de influencia (hinterland) y, en particular, sobre el panorama logístico español ha supuesto un elemento adicional importante para determinadas operaciones de importación/exportación que, hasta el momento, no encontraban el soporte más adecuado.

Los tres actores sobre los que pivota toda la operación de carga aerotransportada son tres: los cargadores, las aerolíneas especializadas, y como interfaz de comunicación, las infraestructuras aeroportuarias. Cada una de estas tres dimensiones forma parte de las variables independientes de la función de oferta, que integran a la totalidad de factores que definirán el precio final de la operación de transporte aéreo.

A nivel internacional, la mejor evolución de la carga aerotransportada se detecta en las líneas de abastecimiento que conectan al Este de Asia con el European Backbone, con la importante emergencia de Oriente Medio como eslabón intermedio de transhipment aéreo.

El análisis de los parámetros de conectividad de la red compleja formada por las conexiones que los nodos aeroportuarios de carga españoles establecen entre sí, y con respecto a los grandes agregados geoeconómicos mundiales, contribuye a clarificar la situación actual del espacio logístico de carga aerotransportada. De este modo, más allá de las cifras anuales de volumen movido de mercancías, es crucial situar a los aeropuertos en su contexto reticular, lo que es posible dada la determinación que el Observatorio del Transporte y la Logística en España, organismo dependiente del Ministerio de Fomento, ha realizado en los nodos aeroportuarios españoles de llegada y de salida a lo largo del período 2004-2014. Esto permite construir una matriz de adyacencia dirigida que incluye el peso en Tm transportado desde el origen al destino.

El análisis cuantitativo de la centralidad de red (grado, cercanía, influencia y autovalor) indica que, a pesar de que, en promedio, el sistema español ha aumentado su volumen neto de mercancías movidas, la conectividad directa (grado) del nodo aeroportuario promedio ha decrecido, de 20 unidades en 2004 a 16 unidades en 2014. Esto no debería de ser preocupante, si la concentración de la actividad logística aerotransportada redundase en una ventaja competitiva, pero el descenso en el resto de parámetros de red (cercanía, influencia y autovalor) indica, claramente, que la pérdida de conexiones directas, en promedio, ha venido acompañada de un importante decremento en la influencia de los nodos, es decir, los hubs importantes han perdido relevancia en la red global de suministro, y los nodos poco conectados no se han significado lo suficiente como para ser calificados de emergentes.

En particular, MAD registra un aumento del 0,7 \% en el volumen de carga movida desde 2004 a 2014, y es el único caso que, habiendo perdido conectividad directa, consigue mantener esa pérdida bajo control. Incluso aumenta ligeramente su capacidad de llegar a otros nodos (cercanía) y su influencia regional. El diagnóstico es, pues, de recuperación sólida. 
$\mathrm{BCN}$, también ha conseguido aumentar en un 1,9\% el volumen de mercancías movido pero, en este caso, la evolución de la centralidad resulta adversa en términos de conectividad directa (-1,5\%), cercanía (-0,3\%) e influencia (-4,0 \%). El diagnóstico es, pues, de una moderada pérdida de centralidad.

El caso de ZAZ reviste más matices en el análisis cuantitativo de sus parámetros de red. La explosión en el volumen de mercancías movido se cifra en un 83,5\% de aumento entre 2004 y 2014. Consigue valores positivos de evolución en la conectividad directa $(0,5 \%)$, y extremadamente favorables en lo que respecta a la evolución de la cercanía (1,1 $\%)$ e influencia regional (25,7 \%). El valor extremo para la evolución de la centralidad de autovalor $(-3,1 \%)$, actúa en este caso como ventaja competitiva, al señalar que la extraordinaria evolución de este aeropuerto de carga se ha realizado, además, con respecto a nodos emergentes con respecto a la cadena global de suministro. El diagnóstico es, claramente, de un puerto con una evolución extremadamente exitosa.

Por último, el nodo de VIT, presenta una evolución adversa en todos los parámetros analizados: en evolución neta del volumen de mercancías movidas (-0,5\%), grado (-2,2 \%), cercanía (-0,1\%), influencia (-0,5\%) y, sobre todo, de centralidad de autovalor $(-2,3 \%)$. En el caso de este último parámetro, los valores negativos de grado, cercanía e influencia necesariamente implican una cierta disfunción en la estrategia comercial de alguno de los actores implicados: cargadores, aerolíneas o infraestructuras aeroportuarias.

El análisis cualitativo de la estructura de red obtenida, una vez aplicado el algoritmo de Kruskal (para determinar las rutas óptimas más importantes en términos de coste) y separado el sistema mediante un algoritmo force-directed, permite complementar el análisis cuantitativo de los parámetros de red. MAD sigue siendo el hub de reparto prioritario para los operadores, atrayendo gran parte de la actividad internacional, excepto en lo referente a los nodos asiáticos, Oriente Medio y los países de la Europa del Este no pertenecientes al espacio Schengen (ex-Yugoslavia, Ucrania, Bielorrusia, Rumanía, Bulgaria y Moldavia), que han desarrollado un vínculo comercial prioritario con ZAZ. La pérdida de influencia de $\mathrm{BCN}$, a favor de $\mathrm{ZAZ}$, se puede apreciar en la total desvinculación de los tráficos asiáticos con respecto a este nodo aeroportuario, operada de modo gradual en los tres años analizados $(2004,2008,2014)$.

En conclusión, las metodologías de análisis de redes complejas, extendidas en otras modalidades logísticas como el transporte por carretera o el transporte marítimo, también proporcionan una visión complementaria a la de la mera contabilidad del volumen anual de mercancías, situando a los aeropuertos de acuerdo a su centralidad, dentro del sistema reticular que conforma la red global de suministro. Queda pendiente, desde un punto de vista científico, la tarea de la integración de todos los modos logísticos para profundizar en mayor medida en la estructura de las funciones de oferta y demanda y, por tanto, en la comprensión del proceso de conformación del precio del transporte.

\section{Referencias}

Abdelghany, K. F., Shah, S. S., Raina, S., \& Abdelghany, A. F. (2004). A model for projecting flight delays during irregular operation conditions. Journal of Air Transport Management, 10(6), 385-394. 
Amaruchkul, K., \& Lorchirachoonkul, V. (2011). Air-cargo capacity allocation for multiple freight forwarders. Transportation Research Part E: Logistics and Transportation Review, 47(1), 30-40.

Barrat, A., Barthelemy, M., Pastor-Satorras, R., \& Vespignani, A. (2004). The architecture of complex weighted networks. Proceedings of the National Academy of Sciences of the United States of America, 101(11), 3747-3752.

Biskup, M. (2011). Graph diameter in long-range percolation. Random Structures \& Algorithms, 39(2), 210-227.

Boeing World Air Cargo Forecast. (2016). Recuperado el 16 de noviembre de 2016, de http://www.boeing.com/commercial/market/cargo-forecast/

Bougheas, S., Demetriades, P. O., \& Morgenroth, E. L. (1999). Infrastructure, transport costs and trade. Journal of International Economics, 47(1), 169-189.

Bourqui, R., Gilbert, F., Sharan, U., Simonetto, P., \& Zaidi, F. (2008). VAST 2008 Challenge: Social network dynamics using cell phone call patterns. In IEEE symposium on visual analytics science and technology.

Bowen, J. T. (2012). A spatial analysis of FedEx and UPS: hubs, spokes, and network structure. Journal of Transport Geography, 24, 419-431.

Cao, J. M., \& Kanafani, A. (2000). The value of runway time slots for airlines. European Journal of Operational Research, 126(3), 491-500.

Carriere, J., \& Kazman, R. (1995). Research report. interacting with huge hierarchies: beyond cone trees. In Information Visualization, 1995. Proceedings. (pp. 7481). IEEE.

Centro de Suministro (2013). El tráfico de mercancías en el aeropuerto de Vitoria. Recuperado de http://www.cadenadesuministro.es/noticias/el-trafico-demercancias-en-el-aeropuerto-de-vitoria-crece-un-76-en-agosto-y-alcanza-las2-497-toneladas/

Chang, Y. H., \& Wang, Y. C. (2010). Significant human risk factors in aircraft maintenance technicians. Safety science, 48(1), 54-62.

Chen, F., Chen, Z., Wang, X., \& Yuan, Z. (2008). The average path length of scale free networks. Communications in Nonlinear Science and Numerical Simulation, 13(7), 1405-1410.

Chi-Lok, A. Y., \& Zhang, A. (2009). Effects of competition and policy changes on Chinese airport productivity: An empirical investigation. Journal of Air Transport Management, 15(4), 166-174.

Chow, C. K. W., \& Fung, M. K. Y. (2009). Efficiencies and scope economies of Chinese airports in moving passengers and cargo. Journal of Air Transport Management, 15(6), 324-329.

Clancy, B., \& Hoppin, D. (2006). Stead climb merger global forecast accelerating intercontinental air freight demand growth through 2010. American Shipper, $7,64-87$ 
Clerot, F. (2015). Do you have a Chu Liu Edmonds Minimum Spanning Tree for Python?. Recuperado de https://www.researchgate.net/post/Do_you_have_a_ Chu_Liu_Edmonds_Minimum_Spanning_Tree_for_Python

CNMC. (2014). Informe de la CNMC sobre el sector aeroportuario español: situación actual y recomendaciones de liberalización. Recuperado de https://www.cnmc. es/Portals/o/Notas \%20de \%20prensa/20140703_Estudio_aeroportuario_ \%20integrado.pdf

Cook, A., Tanner, G., Williams, V., \& Meise, G. (2009). Dynamic cost indexingManaging airline delay costs. Journal of air transport management, 15(1), 2635 .

Coyle, J.J., Bardi, E.J. \& Langley, C.J. (2002). The management of business logistics: a supply chain perspective. Nashvile (TN): Southwestern Publishing Group.

Dillingham, G. L. (2005). Initiatives to reduce flight delays and enhance capacity are ongoing but challenges remain. United States Government Accountability Office

Ducker, M. (2006). Air cargo: enabling access to the global marketplace. XXXIII Air Cargo Forum and Exposition, TIATA, Calgary.

Ducruet, C., \& Notteboom, T. (2012). The worldwide maritime network of container shipping: spatial structure and regional dynamics. Global Networks, 12(3), 395423.

Escalona, A. \& Ramos, D. (2010). Geografía productiva y flujos internacionales: el crecimiento de la carga aérea en el aeropuerto de Zaragoza. Anales de Geografía, $30(2), 59-80$.

Forsyth, P. (2006). Airport competition: regulatory issues and policy implications, in D. Lee (ed.). Advances in Airline Economics. Vol. 1. Competition Policy and Antitrust. Bingley (UK): Emerald Group Publishing Limited

Gardiner, J., Ison, S., \& Humphreys, I. (2005). Factors influencing cargo airlines' choice of airport: An international survey. Journal of Air Transport Management, 11(6), 393-399.

Hesse, M., \& Rodrigue, J. P. (2004). The transport geography of logistics and freight distribution. Journal of transport geography, 12(3), 171-184.

Holten, D., \& Van Wijk, J. J. (2009, June). Force-Directed Edge Bundling for Graph Visualization. In Computer graphics forum (Vol. 28, No. 3, pp. 983-990). New jersey: Blackwell Publishing Ltd.

Hsu, C. I., LIAO, P., YANG, L. H., \& CHEN, Y. H. (2005). High-tech firms'perception and demand for air cargo logistics SERVICES. Journal of the Eastern Asia Society for Transportation Studies, 6, 2868-2880.

IATA Air Cargo. (2016). Recuperado el 16 de noviembre de 2016, de http://www.iata. org/whatwedo/cargo/Pages/index.aspx 
Johns, A. (2012). What is graph density?. Recuperado de https://www.quora.com/ What-is-graph-density

Kruskal, J. B. (1956). On the shortest spanning subtree of a graph and the traveling salesman problem. Proceedings of the American Mathematical society, 7(1), 48-50.

Lambert, A., \& Auber, D. (2012). Graph analysis and visualization with Tulip-Python. In EuroSciPy 2012-5th European meeting on Python in Science.

Lechmann, M. (2011). Economies of scale and scope and its application to the airport industry. Münster: Westfälische Wilhelms Universität

Lee, C., Huang, H. C., Liu, B., \& Xu, Z. (2006). Development of timed Colour Petri net simulation models for air cargo terminal operations. Computers \& industrial engineering, 51(1), 102-110.

Li, Q., Hu, J., Hu, Z., \& Hao, Y. (2015). Development Strategies of the Cargo Transshipment Hub of Shanghai Pudong Airport in Free Trade Zone. In LISS 2014 (pp. 1419-1423). Berlin: Springer

Li, Z., Xu, M., \& Shi, Y. (2015). Centrality in global shipping network basing on worldwide shipping areas. GeoJournal, 8o(1), 47-60.

Limao, N., \& Venables, A. J. (2001). Infrastructure, geographical disadvantage, transport costs, and trade. The World Bank Economic Review, 15(3), 451-479.

Lin, C. C., \& Yen, H. C. (2007). On Balloon Drawings of Rooted Trees. J. Graph Algorithms Appl., 11(2), 431-452.

Lu, H. A., \& Chen, C. Y. (2011). A time-space network model for unit load device stock planning in international airline services. Journal of Air Transport Management, 17(2), 94-100.

Martı́ n, J. C., \& Román, C. (2004). Analyzing competition for hub location in intercontinental aviation markets. Transportation Research Part E: Logistics and Transportation Review, 4O(2), 135-150.

McDonald, R., Pereira, F., Ribarov, K., \& Hajič, J. (2005, October). Non-projective dependency parsing using spanning tree algorithms. In Proceedings of the conference on Human Language Technology and Empirical Methods in Natural Language Processing (pp. 523-530). Association for Computational Linguistics.

Mueller, E. \& Chatterji, G. (2002). Analysis of Aircraft Arrival and Departure Delay Characteristics, AIAA's Aircraft Technology, Integration, and Operations (ATIO) 2002 Technical Forum, doi:10.2514/6.2002-5866

Müller, J., Kamp, V., Niemeier, H. M., Bruche, G., Herr, H., Nagel, F., \& Ripsas, S. (2005). Can we learn from benchmarking studies of airports and where do we want to go from here?. Working Papers No 28, Berlin: Berlin School of Economics and Law, Institute of Management Berlin 
Neibergeer, C. (2008). The effects of deregulation, changed customer requirements and new technology on the organisation and spatial patterns of the air freight sector in Europe. Journal of Transport Geography, 16(4), 247-256.

Newman, M. E. (2006). Modularity and community structure in networks. Proceedings of the national academy of sciences, 103(23), 8577-8582.

Ortega, F. (2016). Gen Air: carga aérea. Recuperado de http://es.slideshare.net/ camaradealava/gen-air-carga-area-francisco-ortega

OTLE (2016). Informe Anual 2015, Madrid.

OTLE (2016b). Consultas de la base de datos y mapas. Recuperado de http:// observatoriotransporte.fomento.es/OTLE/LANG_CASTELLANO/ BASEDATOS/

Pais-Montes, C., Freire-Seoane, M. J., \& Gonzalez-Laxe, F. (2012). General cargo and containership emergent routes: A complex networks description. Transport Policy, 24, 126-140.

Pastor, J.H. (2014). Inditex revoluciona el aeropuerto con otro récord de mercancías. Recuperado de http://www.elperiodicodearagon.com/noticias/temadia/ inditex-revoluciona-aeropuerto-otro-record-mercancias_973556.html

Pejovic, T., Noland, R. B., Williams, V., \& Toumi, R. (2009). A tentative analysis of the impacts of an airport closure. Journal of Air Transport Management, 15(5), 241-248.

Rietveld, P., \& Bruinsma, F. (2012). Is transport infrastructure effective?: transport infrastructure and accessibility: impacts on the space economy. Springer Science \& Business Media.

Rong, A. \& Grunow, M. (2009). Shift design for freight handling personal at air cargo terminals. Transportation Research, part E, 45, 725-739.

Schaefer, L., \& Millner, D. (2001). Flight delay propagation analysis with the detailed policy assessment tool. In Systems, Man, and Cybernetics, 2001 IEEE International Conference on (Vol. 2, pp. 1299-1303). IEEE.

Schmidberger, S., Bals, L., Hartmann, E., \& Jahns, C. (2009). Ground handling services at European hub airports: development of a performance measurement system for benchmarking. International Journal of Production Economics, 117(1), 104-116.

Sharp, W. E., \& Markham, T. (2000). Quasi-symmetry and reversible Markov sequences in sedimentary sections. Mathematical Geology, 32(5), 561-579.

Smyth, M. \& Pearce, B. (2008). Air Travel Demand. IATA Economic Briefing, $n^{o}$ 9, Montreal: IATA

Takebayashi, M. (2011). The runway capacity constraint and airlines' behavior: Choice of aircraft size and network design. Transportation Research Part E: Logistics and Transportation Review, 47(3), 390-400. 
Thelle, M.H. (2012). Airport Competition in Europe. Copenhagen: Copenhagen Economics

van Vliet, N.S.C. (2011). Air cargo alliances: walking on a thin line: a study on the impact of air cargo alliances (tesis). Recuperada de dspace.ou.nl/ bitstream/1820/3705/1/MWNSCvVlietmei2010.pdf

Wong, C. K., \& Wong, S. C. (2002). Lane-based optimization of traffic equilibrium settings for area traffic control. Journal of advanced transportation, 36(3), 349386.

World Bank Group. (2015). Air Transport: annual report. Recuperado de http:// pubdocs.worldbank.org/en/184421457963482031/2015-Air-TransportAnnual-Report-OFVLR-10MAR16.pdf

Wu, C.L. (2005). Inherent delays and operational reliability of airline schedules. Journal of Air Transport Management, 11(4), 273-282.

Wu, Y. (2010). A dual-response forwarding approach for containerizing air cargoes under uncertainty, based on stochastic mixed o-1 programming. European Journal of Operational Research, 207(1), 152-164.

Yaman, H., Karaşan, O. E., \& Pınar, M. Ç. (2001). The robust spanning tree problem with interval data. Operations Research Letters, 29(1), 31-40.

Zhang, A., \& Zhang, Y. (2002). Issues on liberalization of air cargo services in international aviation. Journal of Air Transport Management, 8(5), 275-287.

Zohil, J., \& Prijon, M. (1999). The MED rule: the interdependence of container throughput and transhipment volumes in the Mediterranean ports. Maritime Policy \& Management, 26(2), 175-193. 


\section{Anexo 1}

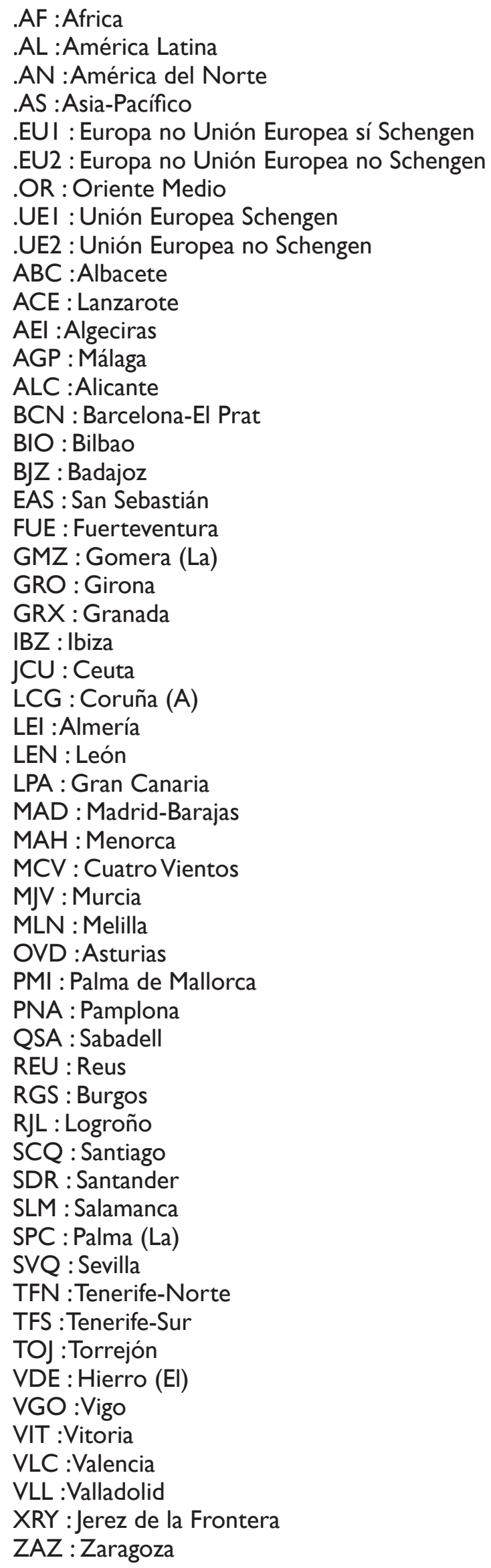




\section{Notas:}

[I] La propuesta de sistemas reticulares heterogéneos en logística del transporte, tal y como se plantea a partir de los datos de OTLE (20l6b) puede resultar controvertida, pero ya hay autores que usan esta metodología de agregación parcial de forma satisfactoria, como Li et al. (2015).

[2] La matriz de adyacencia del grafo $\mathrm{G}^{\mathrm{t}}$, por tanto, es de la forma

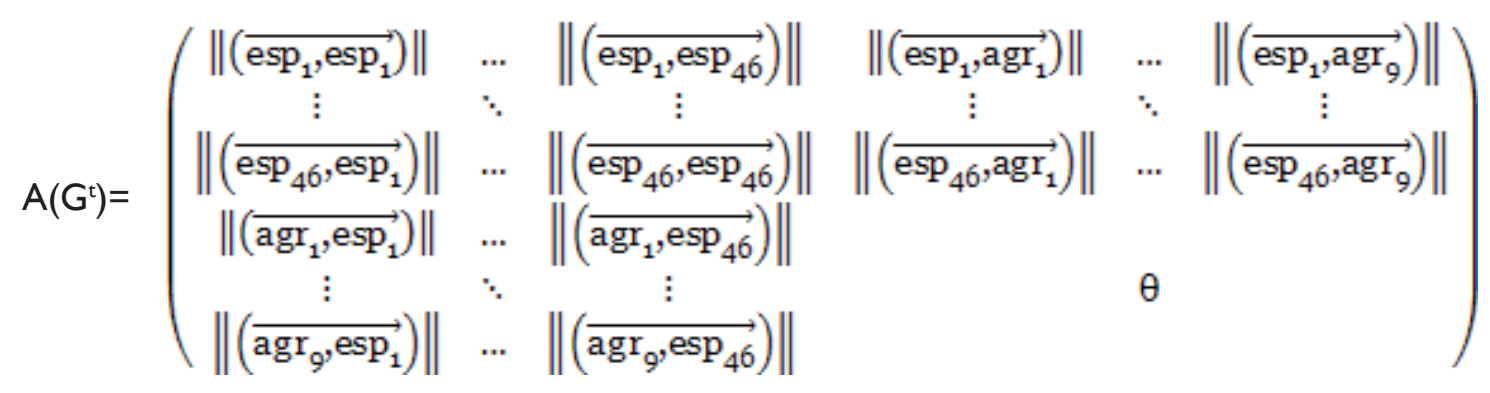

